\title{
BMJ Open eHealth-based intervention to increase physical activity levels in people with cancer: protocol of a feasibility trial in an Irish acute hospital setting
}

\author{
Ciarán Haberlin, ${ }^{\oplus}$ Julie Broderick, ${ }^{1}$ Emer M Guinan, ${ }^{2}$ Catherine Darker, ${ }^{3}$ \\ Juliette Hussey, ${ }^{1}$ Dearbhaile M O'Donnell ${ }^{4}$
}

To cite: Haberlin C, Broderick J, Guinan EM, et al. eHealthbased intervention to increase physical activity levels in people with cancer: protocol of a feasibility trial in an Irish acute hospital setting. BMJ Open 2019;9:e024999. doi:10.1136/ bmjopen-2018-024999

- Prepublication history for this paper is available online. To view these files, please visit the journal online (http://dx.doi. org/10.1136/bmjopen-2017016797).

Received 13 July 2018 Revised 10 January 2019 Accepted 11 January 2019

Check for updates

(C) Author(s) (or their employer(s)) 2019. Re-use permitted under CC BY-NC. No commercial re-use. See rights and permissions. Published by BMJ.

${ }^{1}$ Department of Physiotherapy, University of Dublin Trinity College, Dublin, Ireland

${ }^{2}$ School of Medicine, University of Dublin Trinity College, Dublin, Ireland

${ }^{3}$ Discipline of Public Health \& Primary Care, University of Dublin Trinity College, Dublin,

Ireland

${ }^{4}$ Department of Medical

Oncology, St. James's Hospital, Dublin, Ireland

Correspondence to

Ciarán Haberlin; haberlic@tcd.ie

\section{ABSTRACT}

Introduction Exercise and physical activity (PA) are established and effective treatment options for various side effects of cancer treatments such as surgery, chemotherapy and radiotherapy. The advent of eHealth brings new opportunities to influence healthy behaviours, using interactive and novel approaches. Influencing PA behaviours in people with cancer presents a potential application of this. The aim of this study is to evaluate the feasibility and preliminary efficacy of an intervention, using eHealth, for increasing PA in cancer survivors.

Methods and analysis This will be a single-arm prepost feasibility study. We aim to recruit a heterogeneous sample of 60 participants from cancer clinics in St. James's Hospital, Dublin, Ireland. Eligibility criteria will include patients who have completed chemotherapy and/ or radiotherapy with curative intent between 3 and 36 months prior to enrolment. The intervention will include the delivery of a 12-week PA programme. The eHealth aspect of the intervention will involve the provision of a Fitbit activity tracker, which will be used in conjunction with specific PA goals remotely prescribed and monitored by a physiotherapist. Primary outcomes will be feasibility measures related to the study (recruitment capability, data collection procedures, adherence and compliance, evaluation of the resources to implement the study and evaluation of participant responses to the intervention). Secondary measures will evaluate preliminary efficacy of the intervention in terms of clinical outcomes (body composition, PA (objective and self-report), quality of life and aerobic capacity). Primary and secondary outcomes will be assessed at baseline (as appropriate), at conclusion of the intervention and at a 6-month follow-up.

Ethics and dissemination Ethical approval has been granted by the St. James's Hospital/AMNCH Joint Ethics Committee (2016/05/02). Results from this study will be submitted for publication in peer-reviewed journals, as well as for presentation and dissemination at conferences in the field of oncology and survivorship.

Trial registration NCT03036436; Pre-results.

\section{INTRODUCTION}

Early detection and increasingly effective treatments have led to improved survival rates for cancer. Data from Cancer Research UK
Strengths and limitations of this study

- This study will use an eHealth-focused physical activity (PA) intervention, using commercial Fitbit technology to examine its effectiveness to improve PA behaviours in a clinical population.

- Professional input and support, from a chartered physiotherapist, will be used as a key component of this study.

- Objectively measured PA will be used as an outcome to assess the effectiveness of an eHealth intervention, setting it apart from the current research base in eHealth PA interventions in cancer which have generally adopted self-report PA outcome measures.

- The PA intervention in this study will be grounded in behavioural change science, with a number of behavioural change techniques such as 'self-monitoring of behaviour', 'goal-setting' and 'feedback on behaviour' included in the design of the intervention.

- A limitation is that this study will use a feasibility design, and will therefore not include a control or other comparator arm.

show that half of those diagnosed with cancer in England and Wales survive their disease for 10 years or more (2010-2011). ${ }^{1}$ This reflects a global trend of increasing survival after cancer treatment. The benefits of physical activity (PA) and exercise in patients with cancer have been well documented, with improvements in quality of life (QOL), ${ }^{23}$ function ${ }^{45}$ and some association with a reduced risk of recurrence. ${ }^{67}$ Many cancer treatments, including radiation therapy and chemotherapy, can have longterm effects, which may be ameliorated by exercise. ${ }^{8}$ In spite of these reported benefits, it has been shown that the majority of cancer survivors have difficulty in adhering to healthy lifestyle behaviours, including recommended PA behaviours. ${ }^{9}{ }^{10}$ So far, no single method of exercise promotion has been demonstrated to increase and maintain PA levels in cancer survivors. eHealth, defined by the WHO as 
'the transfer of health resources and healthcare by electronic means', ${ }^{11}$ may present opportunities to address the challenge of improving PA in cancer survivors.

Currently, there are very few studies investigating the use of mobile or wearable technology to increase PA in cancer survivors. In contrast to this, several systematic reviews have been published which primarily focused on eHealth-based PA interventions in community-dwelling adults or in general populations from paediatric to older age groups. ${ }^{12-16}$ The results from those studies demonstrated the effectiveness of their eHealth-based interventions in increasing PA. One of the first systematic reviews which examined the role of technology in promoting PA in a specific disease area was in type II diabetes. ${ }^{17}$ That review also reported increases in PA, highlighting the rationale for incorporating an eHealth component into the promotion of PA in cancer survivors. The inclusion of eHealth in PA interventions, in the form of Fitbit activity tracker utilisation, has also been examined and has also resulted in positive increases in $\mathrm{PA} .^{18}$

We recently completed a systematic review ${ }^{19}$ examining evidence in cancer, according to the Medical Research Council guidelines on developing Complex Interventions. ${ }^{20}$ Our review describes 10 studies which employed eHealth to increase PA in cancer survivors, some of which showed promising results. Eight of ten studies reported statistically significant improvements in PA with the intervention under investigation. However, our review concluded that short- term follow-up, heterogeneity of interventions and lack of agreement about self-report PA measures weakened the interpretability of those studies. Furthermore, the majority were limited by the absence of objective PA measures, using only self-report PA measures. We also recently completed a qualitative focus group study among cancer survivors to explore barriers and facilitators around eHealth PA interventions. These two projects have highlighted the need for, and shaped the design of, an eHealth-based PA intervention which could be scaled up in a full randomised controlled trial (RCT) if feasible and acceptable.

Interventions targeting behavioural change often encompass elements of several behavioural change theories and qualitative studies. Here, aspects of the Social Cognitive Theory $(\mathrm{SCT})^{21}$ and the Transtheoretical Model $^{22}$ have been used to develop the intervention in this study. We also incorporated themes from our recently completed focus group discussions. Behavioural change techniques (as defined by Michie $e t a t^{23}$ ) in the intervention include 'self-monitoring of behaviour', 'goal-setting', 'feedback on behaviour' and 'information about health consequences', among others. These behavioural change techniques have been included as a number are contained within the SCT, which we used to design our study, and also because a number of these techniques were identified by focus group participants as being important in a $\mathrm{PA}$ intervention.

The professional input in this intervention will be delivered by a physiotherapist, an expert in PA promotion and prescription. The input of a professional has been shown to be effective in improving PA levels in patients. ${ }^{24}$ The delivery of this input can take the form of traditional face-to-face contact or remote input, achieved through technology. Both methods of delivery, face to face and remote, have been shown to be effective, ${ }^{24}$ with the challenge now being to identify the optimal blend. This intervention will combine both remote personal healthcare professional input and Fitbit technology in an effort to improve PA in cancer survivors. The study's overall aim is to explore the initial feasibility of that intervention and to provide data required to design a definitive future RCT.

Specific objectives for this study include:

1. To investigate the acceptability to participants of the (a) PA intervention and (b) the Fitbit technology.

2. To provide information needed to design a full-scale RCT including (a) number of participants recruited and dropout rates, (b) suitability of data collection procedures, (c) compliance of participants, (d) resource availability and (e) participant response to the intervention.

3. To assess the preliminary efficacy of this intervention to increase PA, QOL and aerobic capacity and to improve body composition.

\section{METHODS}

\section{Study design}

This feasibility study will use a single-arm longitudinal pre-post test design, with measurements at baseline (T1), 12 weeks (T2) and 24 weeks (+/ -2 weeks) after baseline (T3). The start date for this study was January 2017, with the projected end date being March 2019.

\section{Setting and study participants}

Participants will be identified and recruited from cancer clinics in St. James's Hospital (Dublin, Ireland), a major teaching hospital and the National Bone Marrow Transplant Centre. Clinics include (but are not limited to) the oncology day ward, haematology day ward and follow-up outpatient services, from where we will recruit a heterogeneous sample of participants. Cancer clinicians will be provided with the eligibility criteria for study participation prior to each outpatient clinic, where they will review the patient list and identify suitable candidates for participation. The lead investigator will then liaise with the cancer clinicians to approach those potential participants who expressed an interest to the cancer clinicians, and were deemed eligible for involvement by the clinician. Written informed consent will be gained from each participant prior to inclusion in this study. Inclusion and exclusion criteria are detailed below. The Standard Protocol Items Recommendations for Interventional Trials reporting guidelines were used in preparing this protocol. ${ }^{25}$ The recruitment period for this study is between March 2017 and September 2018, with rolling recruitment used. 


\section{Eligibility criteria}

Eligibility criteria are as follows: (1) agreement of the participant's treating clinician that he/she can participate, including medical clearance to exercise and interest in taking part; (2) aged $>/=18$ years; (3) completed chemotherapy or radiotherapy with curative intent within the preceding 3 years. Participants may have had chemotherapy or radiotherapy as the sole treatment for cancer, with adjunctive surgery, but not surgery alone. Patients who had surgery as part of cancer treatment are eligible, as long as they also had chemotherapy or radiation in the neoadjuvant and/ or adjuvant setting. Participants who are still on adjuvant hormone therapy and/or adjuvant Her2-directed therapy are eligible (with physician agreement as above); (4) able to understand English; (5) owns or has access to a device which is compatible with the Fitbit app, that is, smartphone, tablet or computer. Exclusion criteria include: (1) diagnosis of prostate cancer or upper gastrointestinal cancer (to avoid cross-contamination between exercise studies); patients with these diagnoses are currently being recruited into different disease-specific exercise trials in our centre ${ }^{26}$; (2) chronic medical and orthopaedic conditions that preclude exercise (eg, uncontrolled congestive heart failure or angina, myocardial infarction within 6 months, pulmonary embolism within 3 months, breathing difficulties requiring oxygen use or hospitalisation or osteoarthritis causing significant mobility impairment); (3) confirmed pregnancy; (4) dementia, cognitive impairment or psychiatric illness that would preclude ability to participate in the study; (5) incomplete haematological recovery after chemotherapy (WCC $<3, \mathrm{Hb}<10$ or platelets $<100$ ); (6) patients $<18$ years; and ( 7$)$ evidence of active cancer.

\section{Procedure}

The lead researcher will meet potential participants at their outpatient appointment, where they will be provided with a consent form, participant information leaflet and a verbal explanation of the study. Participants who are willing to take part in the study at that point will sign a consent form and be given an ActiGraph PA monitor to bring home. After a 2-day cooling off period, the researcher will contact them. If the participants confirm continued willingness and availability to participate in the study, they will be instructed to wear the ActiGraph for 7 days. Potential participants who do not sign the consent form at the first meeting, but would like to consider participation, will take the consent form and information leaflet and will be instructed to send the signed consent form back to the lead researcher when they have had sufficient time to consider the study. Those participants will be sent the ActiGraph activity monitor (in a padded envelope using the national postal service) once the signed consent form has been received by the researcher, prior to their baseline session.

\section{Baseline session}

Eligible participants will attend a baseline session, with a chartered physiotherapist who is also a study researcher. This session will consist of three components: baseline study measurements, participant evaluation and setting of appropriate individual PA goals and education. The baseline measurements are described in efficacy outcomes below. The physiotherapist will decide on and set appropriate PA goals for the start of the 12-week intervention using a subjective assessment of the participant's current PA level, as described by the Godin leisure time questionnaire and a subjective evaluation on interview of the participant's goals and PA preferences. The physiotherapist is blinded to the results of the baseline ActiGraph PA results at that point. The educational session will include an information session on PA following cancer treatment. Participants will also be introduced to the technological component of the study. Each participant will be given a Fitbit and instructions on how to download the paired smartphone application. If a participant is unfamiliar with this type of technology, a family member will also be invited to receive the training. Participants will be instructed to wear the Fitbit on their waist, bra or in their pocket, depending on their preference. They will also be instructed to wear the Fitbit at all times during their waking hours, apart from when it is not appropriate, such as when going for a shower.

\section{Intervention}

Following the baseline session, participants will wear the Fitbit and upload their PA data. A physiotherapist researcher who can access the study identity codes will review the data. The physiotherapist and the participant will have shared access to a study-specific Fitbit account, with $\log$ in details shared. This review of the data will allow the physiotherapist to monitor the participant's progress towards their goals, specifically their daily step count and their weekly moderate intensity exercise bouts. Participants will each receive scheduled calls, the frequency of which will be tapered on a phased basis throughout the intervention. These phone calls will be delivered by a chartered physiotherapist, an expert in prescribing exercise and physical activity goals. Participants will receive two calls each week until week 4 , one call a week for the next 4 weeks and a call once every fortnight in the last 4-week period. The content of the calls is specific and designed around behavioural change elements. The study personnel will provide participants with feedback and an update on goals based on the PA data, as well as a reminder and advice on uploading Fitbit data. The goals prescribed by the physiotherapist will be collaborative in nature, with participants encouraged to provide feedback about their ability to achieve their goals and any changes they would like to these goals moving forward. Ongoing technological support regarding the Fitbit will also be provided to participants in these phone calls if required. If participants inform the study team that they will be uncontactable 
by telephone, for example, abroad, for some of these scheduled phone calls, we will request permission to contact them during those times via a secure messaging service, to allow for an uninterrupted schedule of reminders. If a participant is unwilling to complete the intervention, and requests to be discontinued, the lead researcher will stop the intervention for this particular participant.

This study will use the commercially available Fitbit wearable technology with its paired smartphone application. The two components will work in tandem to measure PA and, it is hoped, to motivate participants to become more active physically. We chose the 'Fitbit One', a three-dimensional accelerometer which can track daily activity including steps taken, distance travelled and active minutes. Goal setting is also an important feature of this device, as the paired smartphone application allows participants to view their progress, record their workouts and $\log$ food intake. It also wirelessly uploads data to a website that provides graphical visualisations of daily activity patterns for participants to view. For this study, with consideration for the cancer population, PA goals will be individually prescribed by a qualified, chartered physiotherapist using the American Cancer Society guidelines. ${ }^{27}$ Goals for this study will include daily step goals and also weekly moderate intensity exercise goals, and will be tailored to each participant. Participants will use the Fitbit and its paired application for the 12 weeks of the intervention. They will return the Fitbit at 12 weeks post baseline. A study-specific Fitbit account (on Fitbit. com) and password will be created for each participant. With participants' permission, the investigators will have access to participants' activity data throughout the course of the intervention. Participants will also be encouraged to monitor their own daily PA using the Fitbit application.

\section{Feasibility outcomes}

Feasibility outcomes will be assessed at intervention completion.

- Evaluation of recruitment capability and resulting sample characteristics: The number of participants we can recruit will be assessed. Recruitment strategies used will be assessed for future potential trials in this area.

- Evaluation and refinement of data collection procedures and outcome measures: Procedures to collect data will be assessed for the potential burden of time for the participant. This will include calculating the time taken for all assessment time-points, while also accounting for the time taken by receiving all calls in the phone call schedule.

- Evaluation of the adherence and compliance of the intervention and study procedures: Compliance with complete synchronisation and wear time each week for the Fitbit and adherence to the intervention duration will be assessed. Adherence will be measured by attendance at baseline and exit session, with further details on compliance gained from information on goal attainment and 'attending' the phone call schedule.

- Evaluation of the resources and ability to manage and implement the study and intervention: The ability of study personnel to implement the study with available resources will be assessed.

- Preliminary evaluation of participant responses to intervention: The outcome will be measured qualitatively by inviting participants to provide feedback on the intervention's likelihood of being successful. Satisfaction with the technological intervention will be measured using a participant questionnaire at study end (T2), which will assess any difficulties the participants may have with the intervention and also what they liked about using it.

\section{Efficacy outcomes}

Efficacy outcomes will be measured at T1, T2 and T3. Below are the outcomes and their methods of measurement in detail.

- Body composition: Anthropometric parameters will be measured at T1, T2 and T3. Bioelectrical impedance analysis will be conducted using a Seca device. This will be measured in percentage body fat.

- Body weight: This will be measured using a standardised digital scale. Standing height will be measured, without shoes, to the nearest millimetre $(\mathrm{mm})$ using a stadiometer.

- Body mass index: This will be calculated by dividing weight in kilograms by height in metres squared.

- Waist circumference: This will be measured according to standard methods.

- PA: Description of all accelerometer settings for data collection and analysis are detailed below. This study will follow recommendations for reporting of accelerometer data by Montoye et al. ${ }^{28}$ The ActiGraph GT3X-BT triaxial accelerometer will be used to monitor 7 days of activity at time-points T1, T2 and T3. There is a mandatory 3-month follow-up period from intervention end until T3, but we will retain the option to invite participants back for a further ActiGraph measurement 3 months after T3, to explore efficacy 6 months from intervention end. The ActiGraph measures activity in three dimensions (vertical (x), antero-posterior (y) and medio-lateral (z)) and generates a summary variable-vector magnitude. Time spent sedentary will be measured by the accelerometer also. Epoch length for data collection will be $1 \mathrm{~s}$, while epoch length for data analysis will be 60 s. Participants will be instructed to wear the ActiGraph on their waist, and will also be instructed to record non-wear time of the ActiGraph in a daily log. The ActiGraph will be distributed either in person or through the national postal service. The number of valid days and number of minutes per day of accelerometer data needed to be included in analysis is a wear time of $>4$ days with $>10$ hours of wear time per day, including at least one weekend day. This was 
determined using an algorithm devised by Choi et $a l .{ }^{29}$ The PA outcome of interest in this study is the time spent in moderate-to-vigorous PA. We will use Freedson et al cut points to analyse and interpret this outcome. ${ }^{30}$ Sedentary time will also be investigated, with cut points defined by Troiano et al used to interpret this outcome. ${ }^{31}$ Raw data from the monitors will be processed by ActiLife software (V.6 13.3).

- Self-report PA: We will use the modified version of the Godin Leisure Time Exercise Questionnaire which has been shown to be a reliable and valid self-report measure of $\mathrm{PA}^{32}$ The questionnaire contains three questions that assess the average frequency and duration of mild, moderate and strenuous exercise during free time in a typical week. Godin et al reported the test-retest reliability coefficient of the Godin questionnaire to be 0.64 and the concurrent validity to range from 0.38 to 0.54 over three validity criteria. ${ }^{32}$

- QOL: We will use the Functional Assessment of Cancer Therapy (FACT-G) scale (general) ${ }^{33}$ and the physical functional measure of the SF-36 (Short-Form Health Survey). ${ }^{34}$ A statistically significant increase of greater than 4.0 points on the FACT scale represents a clinically meaningful improvement in QOL from exercise. ${ }^{35}$ The FACT-G (V.4) is a 27 -item questionnaire divided into four primary QOL domains: physical well-being, social/family well-being, emotional well-being and functional well-being and an overall QOL level is also yielded. This questionnaire was initially developed by Cella $e t a l^{33}$ and has been validated in a mixed cancer population. Strong concurrent validity is reported by strong Pearson correlations with the Functional Living Index-Cancer (0.79) and the patient-completed version of the Quality of Life (QL) index (0.74). Using the global rating of change scale as an anchor, Cella et al proposed that a clinically meaningful change corresponds to a total FACT-G raw score in the range of $5-7$ points. ${ }^{35}$ A 2008 review by Victorson $e t a l^{36}$ reported the average reliability of the FACT-G to be 0.88 with the reliability of subsets ranging from 0.71 to 0.83 . The SF-36v2 is a widely used generic measure of health status. Psychometric properties of the SF-36 have been well established. ${ }^{37}$ Thirty five of the 36 items are grouped into eight scales that address health constructs considered to be important to most healthcare situations: physical functioning, role limitations (physical problems), bodily pain, general health, vitality, social functioning, role limitations (emotional problems) and mental health. We will use the Physical Health measure only of the SF-36 for the purposes of this study.

- Aerobic capacity/endurance: This will be measured using the 6 min walk test $(6 \mathrm{MWT})^{38}$ at T1, T2 and T3. This will be conducted in a dedicated clinical research facility with a qualified physiotherapist. The 6MWT was shown to be valid and reliable in patients with cancer by Schmidt et al. ${ }^{39}$
Sample size

The difficulties of generating accurate sample size calculation for feasibility studies are well known. For feasibility studies, sample sizes between 24 and 50 have been recommended.$^{40}$ Based on this, we propose to recruit a sample size of 60 which allows for a $20 \%$ dropout.

\section{Statistical approach}

Data will be tested for normality and homogeneity of variances using the Shapiro-Wilk test and Mauchly's Test of Sphericity. Friedman's test will be used to compare non-normally distributed data, and analysis of variance (ANOVA) repeated measures parametric tests will be used to compare normally distributed data for each of the stated outcomes at each time-point. If deemed necessary, multivariate analysis, including multi-factorANOVA and multiple regression, will be conducted to adjust potential confounders, such as baseline demographic and PA levels. Statistical Package for the Social Sciences V.22 (IBM Corp., Armonk, New York, USA) will be used for all analyses and the significance level will be set at 0.05 .

\section{Participant withdrawal from study and/or from follow-up}

Non-retention rate, when participants withdraw consent or are lost to follow-up so outcome data cannot be obtained, will be recorded. If participants are happy to give a reason for their withdrawal from the study, this will be documented. Non-adherence, when participants deviate from the intervention but provide follow-up assessment, will also be recorded, as will any adverse events that occur throughout the intervention.

\section{Data management}

All forms relating to study data will be anonymised and kept in locked cabinets. Individual participant clinical data and study results will be added to a password-protected spreadsheet for analysis. The spreadsheet will contain only anonymised data using the study serial number and not the patient hospital number. The date of birth will be used to calculate age but only age will appear on the spreadsheet. Results will be analysed together and coded anonymously throughout. Two copies of a coded list of participants will be stored in separately located, locked filing cabinets. It was decided that a data monitoring committee was not required for this study, and no interim analysis is planned.

\section{Patient and public involvement}

Patient experience and input was central to the development of this study. The literature review conducted initially was followed by two separate scoping studies, each a qualitative research study aiming to collect and assimilate patient opinions and experiences to aid in the design of this trial, the Improving Physical Activity and Exercise with Technology Use in Survivors of Cancer (IMPETUS) trial. All participants recruited for those two studies were cancer survivors. The first study was a questionnaire-based study which consisted of 10 
questions. Within the questions, we hoped to address three main topics, specifically the PA status and smartphone usage of participants as well as interest in participating in further studies. The second study took the form of a series of focus groups, as mentioned above. The focus groups explored perceptions of the possible use of eHealth to increase physical activity as well as mapping facilitators and barriers to the possible use of eHealth to increase PA. The valuable input we received from these patients helped in developing this current study. Both of these studies are being written up for publication at this time.

It is planned that results of this trial will be disseminated to participants by a newsletter drawn up by the lead researcher with all final results and conclusions included. This will then be posted to participants. Patients were not formally involved in the recruitment to this study.

\section{ETHICS AND DISSEMINATION}

Written, informed consent will be required from each participant prior to taking part in the study. In addition to this, all participants must have agreement from their oncology clinician to take part. Ethical approval for this trial has been granted. This study will also adhere to the recent General Data Protection Regulation guidelines. The lead investigator will have access to the final trial data set. Results from this study will be submitted for publication in peer-reviewed journals. Findings from this study will also be submitted for presentation at conferences in the field of cancer and survivorship.

Acknowledgements We would like to extend a special thank you to all the input of patients from St. James's Hospital, Dublin that were involved in the development of this study.

Contributors $\mathrm{CH}, \mathrm{DMO}$ 'D, JB, CD, EMG and JH contributed to the design and structure of this research. $\mathrm{CH}, \mathrm{DMO}$ 'D and JB wrote and prepared the manuscript.

Funding This research received no specific grant from any funding agency in the public, commercial or not-for-profit sectors.

Competing interests None declared.

Patient consent for publication Not required.

Ethics approval St. James's Hospital/AMNCH joint ethics committee (Reference number: 2016/05/02)

Provenance and peer review Not commissioned; externally peer reviewed.

Open access This is an open access article distributed in accordance with the Creative Commons Attribution Non Commercial (CC BY-NC 4.0) license, which permits others to distribute, remix, adapt, build upon this work non-commercially, and license their derivative works on different terms, provided the original work is properly cited, appropriate credit is given, any changes made indicated, and the use is non-commercial. See: http://creativecommons.org/licenses/by-nc/4.0/.

\section{REFERENCES}

1. Cancer Research UK. Cancer research. http://www. cancerresearchuk.org/health-professional/cancer-statistics-for-theuk

2. Adamsen L, Quist M, Andersen C, et al. Effect of a multimodal high intensity exercise intervention in cancer patients undergoing chemotherapy: randomised controlled trial. BMJ 2009;339:b3410.
3. Mutrie N, Campbell AM, Whyte F, et al. Benefits of supervised group exercise programme for women being treated for early stage breast cancer: pragmatic randomised controlled trial. BMJ 2007;334:517.

4. Morey MC, Snyder DC, Sloane R, et al. Effects of home-based diet and exercise on functional outcomes among older, overweight longterm cancer survivors: RENEW: a randomized controlled trial. JAMA 2009;301:1883-91.

5. Courneya KS, Friedenreich CM, Sela RA, et al. The group psychotherapy and home-based physical exercise (group-hope) trial in cancer survivors: physical fitness and quality of life outcomes. Psychooncology 2003;12:357-74.

6. Friedenreich CM, Gregory J, Kopciuk KA, et al. Prospective cohort study of lifetime physical activity and breast cancer survival. Int J Cancer 2009;124:1954-62.

7. Holmes MD, Chen WY, Feskanich D, et al. Physical activity and survival after breast cancer diagnosis. JAMA 2005;293:2479-86.

8. Schmitz KH, Courneya KS, Matthews $\mathrm{C}$, et al. American college of sports medicine roundtable on exercise guidelines for cancer survivors. Med Sci Sports Exerc 2010;42:1409-26.

9. DeNysschen C, Brown JK, Baker M, et al. Healthy Lifestyle Behaviors of Breast Cancer Survivors. Clin Nurs Res 2015;24:504-25.

10. Blanchard CM, Courneya KS, Stein K. Cancer survivors' adherence to lifestyle behavior recommendations and associations with healthrelated quality of life: results from the American Cancer Society's SCS-II. J Clin Oncol 2008;26:2198-204.

11. World Health Organization. WHO Telemedicine: opportunities and developments in Member States: report on the second global survey on eHealth. Geneva, Switzerland: World Health Organization, 2009.

12. Norman GJ, Zabinski MF, Adams MA, et al. A review of eHealth interventions for physical activity and dietary behavior change. Am J Prev Med 2007;33:336-45.

13. Krebs P, Prochaska JO, Rossi JS. A meta-analysis of computertailored interventions for health behavior change. Prev Med 2010;51:214-21.

14. Davies CA, Spence JC, Vandelanotte C, et al. Meta-analysis of internet-delivered interventions to increase physical activity levels. Int J Behav Nutr Phys Act 2012;9:52.

15. Foster $\mathrm{C}$, Richards J, Thorogood M, et al. Remote and web 2.0 interventions for promoting physical activity. Cochrane Database Syst Rev 2013;9:Cd010395.

16. Aalbers T, Baars MA, Rikkert MG. Characteristics of effective Internet-mediated interventions to change lifestyle in people aged 50 and older: a systematic review. Ageing Res Rev 2011;10:487-97.

17. Connelly J, Kirk A, Masthoff J, et al. The use of technology to promote physical activity in Type 2 diabetes management: a systematic review. Diabet Med 2013;30:1420-32.

18. Cadmus-Bertram LA, Marcus BH, Patterson RE, et al. Randomized trial of a fitbit-based physical activity intervention for women. Am J Prev Med 2015;49:414-8.

19. Haberlin C, O'Dwyer T, Mockler D, et al. The use of eHealth to promote physical activity in cancer survivors: a systematic review. Support Care Cancer 2018:3323-36.

20. Craig P, Dieppe P, Macintyre S, et al. Developing and evaluating complex interventions: the new medical research Council guidance. BMJ 2008;337:a1655.

21. Bandura A. Self-efficacy: toward a unifying theory of behavioral change. Psychol Rev 1977;84:191-215.

22. Prochaska JO, Velicer WF. The transtheoretical model of health behavior change. Am J Health Promot 1997;12:38-48.

23. Michie S, Richardson $\mathrm{M}$, Johnston $\mathrm{M}$, et al. The behavior change technique taxonomy (v1) of 93 hierarchically clustered techniques: building an international consensus for the reporting of behavior change interventions. Ann Behav Med 2013;46:81-95.

24. James EL, Ewald BD, Johnson NA, et al. Referral for expert physical activity counseling: a pragmatic RCT. Am J Prev Med 2017;53:490-9.

25. Chan AW, Tetzlaff JM, Altman DG, et al. SPIRIT 2013 statement: defining standard protocol items for clinical trials. Ann Intern Med 2013;158:200-7.

26. Sheill G, Brady L, Guinan E, et al. The ExPeCT (Examining Exercise, Prostate Cancer and Circulating Tumour Cells) trial: study protocol for a randomised controlled trial. Trials 2017;18:456.

27. Kushi LH, Doyle C, McCullough M, et al. American Cancer Society Guidelines on nutrition and physical activity for cancer prevention: reducing the risk of cancer with healthy food choices and physical activity. CA Cancer J Clin 2012;62:30-67.

28. Montoye AHK, Moore RW, Bowles HR, et al. Reporting accelerometer methods in physical activity intervention studies: a systematic review and recommendations for authors. $\mathrm{Br} J$ Sports Med 2018;52:1507-16. 
29. Choi L, Liu Z, Matthews CE, et al. Validation of accelerometer wear and nonwear time classification algorithm. Med Sci Sports Exerc 2011;43:357-64.

30. Freedson PS, Melanson E, Sirard J. Calibration of the computer science and applications, inc. accelerometer. Med Sci Sports Exerc 1998;30:777-81.

31. Troiano RP, Berrigan D, Dodd KW, et al. Physical activity in the United States measured by accelerometer. Med Sci Sports Exerc 2008;40:181-8.

32. Godin G, Shephard RJ. A simple method to assess exercise behavior in the community. Can J App/ Sport Sci 1985;10:141-6.

33. Cella DF, Tulsky DS, Gray G, et al. The functional assessment of cancer therapy scale: development and validation of the general measure. J Clin Oncol 1993;11:570-9.

34. McHorney CA, Ware JE, Raczek AE. The MOS 36-Item Short-Form Health Survey (SF-36): II. Psychometric and clinical tests of validity in measuring physical and mental health constructs. Med Care 1993;31:247-310.
35. Cella D, Eton DT, Lai JS, et al. Combining anchor and distributionbased methods to derive minimal clinically important differences on the Functional Assessment of Cancer Therapy (FACT) anemia and fatigue scales. J Pain Symptom Manage 2002;24:547-61.

36. Victorson D, Barocas J, Song J, et al. Reliability across studies from the functional assessment of cancer therapy-general (FACT-G) and its subscales: a reliability generalization. Qual Life Res 2008;17:1137-46.

37. Ware JE, Snow KK, Kosinski M, et al. SF-36 health survey. Manual and interpretation guide. Boston: The Health institute, New England Medical Center, 1993.

38. ATS Committee on Proficiency Standards for Clinical Pulmonary Function Laboratories. ATS statement: guidelines for the six-minute walk test. Am J Respir Crit Care Med 2002;166:111-7.

39. Schmidt K, Vogt L, Thiel C, et al. Validity of the six-minute walk test in cancer patients. Int J Sports Med 2013;34:631-6.

40. Sim J, Lewis M. The size of a pilot study for a clinical trial should be calculated in relation to considerations of precision and efficiency. $J$ Clin Epidemiol 2012;65:301-8. 